\title{
Tecnología lítica y uso diferencial del espacio en Estancia La Suiza, San Luis, Argentina
}

\author{
Gisela Sario* \\ *IDACOR-CONICET, Museo de Antropología, Facultad de Filosofía y Humanidades, \\ Universidad Nacional de Córdoba, Argentina. E-mail: giselasario@hotmail.com
}

RMA

Arqueología

\begin{abstract}
Resumen
El trabajo pretende contribuir, desde la perspectiva de la organización de la tecnología, al conocimiento de las ocupaciones cazadoras-recolectoras que habitaron las inmediaciones de la Sierra de la Estanzuela, en la provincia de San Luis, Argentina.

Se explican cómo fueron las secuencias de producción de herramientas en los sitios de la localidad arqueológica Estancia La Suiza, a partir del análisis de los materiales líticos recuperados en excavación y prospecciones sistemáticas. Las metodologías propuestas para abordar este estudio corresponden al análisis nodular y más específicamente a una combinación del análisis tipológico y no tipológico.

Los resultados permiten interpretar que en esta localidad hubo un uso diferencial del espacio, particularmente en dos sitios que son Estancia La Suiza 2 y Estancia La Suiza 3.
\end{abstract}

Palabras clave: cazadores-recolectores; tecnología lítica; análisis no tipológico; uso diferencial del espacio; San Luis.

Lithic technology and differential use of space in Estancia La Suiza, San Luis, Argentina

\begin{abstract}
This paper aims at gaining insight, from the angle of technological organization, into the hunter-gatherer communities that dwelled in the surrounding areas of Sierras de la Estanzuela, in the province of San Luis, Argentina.

The sequence of tool development in the archaeological site of Estancia La Suiza is described from analysis of lithic materials from excavation and systematic surveys. The methods adopted for conducting this study form part of Minimal Nodule analysis (MANA) and more specifically a combination of typological and non-typological analysis. Results indicate that this site evidences a differential use of space, more specifically found in Estancia La Suiza 2 and Estancia La Suiza 3.
\end{abstract}

Keywords: hunter-gatherers; lithic technology; non typological analysis; differential use of space; San Luis.

El objetivo central de este trabajo es dar a conocer cómo fueron las secuencias de producción de instrumentos en la localidad arqueológica Estancia La Suiza. El interés surge a partir de que la localidad, ubicada en el Noreste de la provincia de San Luis (Argentina) y cercana al faldeo occidental de la sierra de Comechingones, presenta una serie de sitios al aire libre en los cuales se recuperaron materiales líticos mediante prospecciones sistemáticas y una excavación estratigráfica (Laguens et al. 2007, 2009; Sario 2007, 2009, 2011). Entre los materiales hallados se encuentran núcleos, artefactos formatizados y desechos de talla. En estos últimos se va a enfocar el análisis lítico bajo dos perspectivas, la tipológica (Aschero 1983) y otra denominada "no tipológica" (Non Typological Approach) por Ingbar et al. (1989), la cual permite mediante la utilización de unos pocos atributos situar a las lascas dentro de una trayectoria de producción.

En general, para contextos de cazadores recolectores, los desechos líticos son recuperados en las excavaciones sistemáticas en grandes cantidades, y es recién en las últimas décadas que han sido mayormente incluidos en los estudios analíticos. Siguiendo a Larson (2004), con el crecimiento de los estudios de detalle en el registro arqueológico, los desechos de talla se han convertido en un significante objeto de estudio con importantes desarrollos teóricos-metodológicos sobre todo con el advenimiento de la Nueva Arqueología en Estados Unidos (Alher 1989; Amick y Mauldin 1997; Eerkens et al. 2007; Ingbar 1994; Larson y Kornfeld 1997; Magne 2001; Shott y Sillitoe 2005; Sullivan y Rozen 1985, por citar algunos). En Argentina, el análisis de estos artefactos también ha ofrecido información relevante para comprender y explicar los procesos de manufactura de la roca (Bellelli et al. 1985-87; Cattáneo 2006, Charlin 2009; Escola 2000; González de Bonaveri y Horovitz 1991; Espinosa 1998; Flegenheimer 1991; Nami y Bellelli 1994; Valverde 2003, entre otros). 
Los estudios experimentales de talla lítica también le han otorgado un lugar importante a los desechos, en las que el estudio analítico ha permitido resolver algunos problemas o preguntas de los arqueólogos (Amick et al. 1989; Bradbury y Carr 1995; Nami 1997-1998, 2007; Prentiss 1998, entre otros).

En este caso de estudio, para la aplicación del análisis no tipológico a los conjuntos líticos, es necesario previamente haber aplicado otro método, el análisis nodular o MANA (Larson y Kornfeld 1997), el cual permite determinar un número mínimo de eventos de talla.

\section{La Metodología de Análisis Nodular o MANA}

En el estudio de los conjuntos líticos se ha buscado una metodología que permita reconstruir los eventos de talla, entendiendo a éstos como procesos de reducción de núcleos, clastos o la manufactura de un artefacto. El análisis nodular fue propuesto en 1997 por Mary Lou Larson y Marcel Kornfeld y consiste en establecer, mediante el agrupamiento, números mínimos de artefactos que pudieran haber pertenecido al mismo evento de talla. Para ello, se debe tener en cuenta ciertos rasgos petrológicos, como el color, la textura, presencia de inclusiones, etc. Estos autores en función de la composición del número artefactos en cada nódulo o evento, los dividen en simples o múltiples. Los simples son aquellos que contienen sólo un artefacto, y los múltiples, aquellos de dos o más artefactos. En base a las diferentes combinaciones artefactuales se discuten las actividades que cada uno de ellos representa (para discusiones en torno a este tema ver Cattáneo 2005, 2006; Larson 2004; Larson y Finley 2004; Larson y Kornfeld 1997; Sario 2009, 2011).

\section{El Análisis No Tipológico, Tipológico y los Estudios Experimentales}

El análisis no tipológico fue planteado por Ingbar, Larson y Bradley en 1989 y consiste en una serie de modelos que permiten evaluar el grado de reducción de los desechos de talla durante un proceso continuo de producción de artefactos. Mediante una serie de experimentos de talla lítica realizadas por Bradley, en función de diferentes materias primas, estos autores recurrieron a fórmulas matemáticas que permitieran posicionar los desechos a lo largo de una trayectoria de reducción. Uno de estos modelos, el cuatro, es el que presenta el grado de confianza más elevado $(0,94)$ y por lo tanto, es el seleccionado en este análisis. El modelo considera como variables el tamaño, el espesor y la cantidad de lascados en la cara dorsal del desecho entero (para discusiones en torno a este tema ver Cattáneo 2005, 2006; Larson 2004; Larson y Finley 2004; Sario 2009, 2011). De esta manera, se calcula la superficie, la densidad de lascados dorsales, el logaritmo del espesor, el logaritmo de la densidad de lascados dorsales y el logaritmo de la superficie de los desechos de talla pertenecientes al mismo nódulo o evento. La finalidad es que permite determinar cuáles fueron los momentos, dentro de la secuencia continua de talla, a los cuales corresponderían estos desechos.

Por otra parte, en el análisis de los mismos como indicadoras de reducción, consideramos al tamaño como una medida que es adecuada para identificar los momentos de reducción. Los tamaños de desechos enteros son definidos siguiendo la tipología de Aschero (1983), en donde los tamaños se definen en muy pequeño (1), pequeño (2), mediano-pequeño (3), mediano-grande (4), grande (5), muy grande (6) y grandísimo (7). En un proceso de reducción es esperable que los mayores tamaños estén indicando los momentos iniciales y los menores tamaños los momentos finales.

Otra de las variables consideradas es la cantidad de lascados previos en la cara dorsal, que de acuerdo a su número también nos podría estar indicando momentos iniciales o finales. Por lo tanto, la menor cantidad de negativos representan momentos iniciales de producción, y la mayor cantidad, momentos finales.

Algunas ventajas del método no tipológico es que es rápido de aplicar y sólo se trabaja con unas pocas variables que son cuantitativas, lo cual reduce los errores interobservador, pudiéndose comparar conjuntos líticos que proceden de distintos investigadores.

\section{Estancia La Suiza 2 (ELS 2)}

Este sitio es una cantera taller (ELS2) (Laguens et al. 2007) ubicada en el sector central de la Sierra de la Estanzuela. Allí mismo, se encuentra una falla tectónica que es recorrida por el arroyo El Carrizal, de carácter permanente en algunos tramos y en las cercanías hay una explotación de caliza de tiempos recientes, en las que se han conservado sectores sin perturbación de la sierra.

En el lugar se realizaron prospecciones sistemáticas, cuyos resultados permitieron la identificación de distintos tipos de afloramientos, gneises, granitos, esquistos, cuarzos y brechas silíceas, los cuales han tenido un uso potencial para los grupos humanos del pasado. Son de particular interés las rocas silíceas, las cuales presentan diferentes variedades y son aptas para la talla lítica. En las inmediaciones de éstas se realizaron cuatro cuadrículas de recolección en donde se recuperó material de superficie.

\section{Estancia La Suiza 3 (ELS 3)}

Es un sitio taller al aire libre (Laguens et al. 2009, Sario 2009) que se encuentra a unos 50 metros del arroyo El Carrizal y a 700 m de ELS 2 (Figura 1). Se procedió a realizar una excavación sistemática por el hallazgo de materiales líticos que quedaban al descubierto por fuertes procesos 


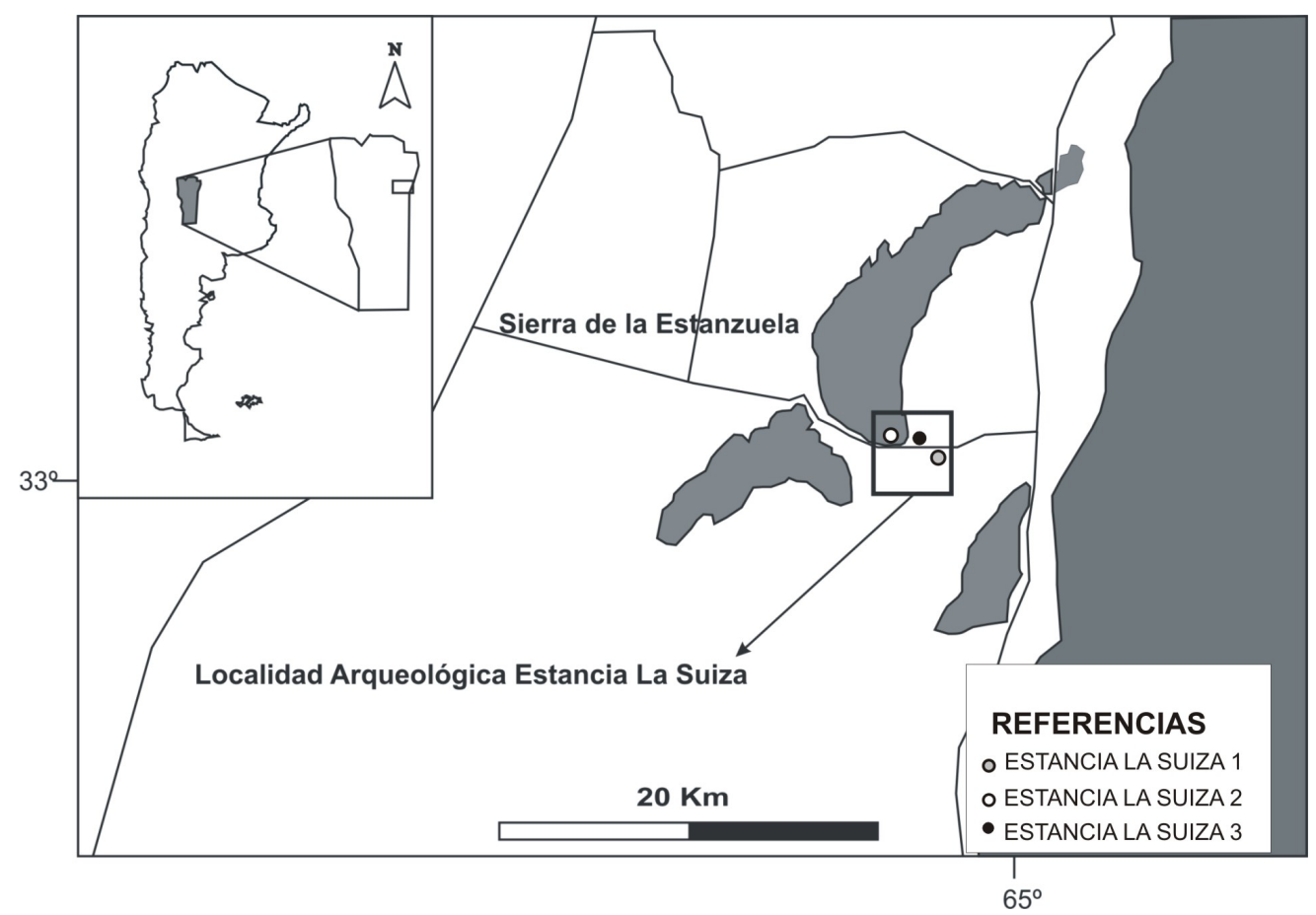

Figura 1. Ubicación de los sitios ELS 2 y ELS 3 de la localidad Estancia La Suiza

Figure 1. Location of sites ELS 2 and ELS 3 of the Estancia La Suiza locality.

erosivos en la zona. Se determinaron dos componentes, el primero contiene tres unidades estratigráficas, la once, catorce y dieciséis que corresponden al componente I, que es el más antiguo de la excavación, y el segundo contiene dos unidades estratigráficas, la dos y la siete, que pertenecen al componente II, más reciente. No posee fechados absolutos. Se realizaron dos intentos de datación, el primero con el único resto óseo recuperado de la excavación, que no contenía suficiente colágeno, y el otro con restos de carbón que no poseían el peso necesario para datar.

\section{El Análisis Lítico}

Como primer paso al estudio del material lítico se aplica el análisis nodular a cada una de las cuatro recolecciones, en donde la totalidad del conjunto lítico recuperado en ELS 2 (cantera) contiene 204 artefactos. De esta manera, se pudieron determinar nueve nódulos, tres simples y seis múltiples. En la conformación de los nódulos múltiples se observan dos nódulos de núcleos y desechos, dos grupos de desechos de talla, un nódulo de artefactos formatizados y desechos, y por último, un grupo de núcleos, artefactos formatizados y desechos (Sario 2011).

La materia prima es casi en su totalidad roca silícea, a excepción de un artefacto de cuarzo y el $66 \%$ presenta corteza.

En cuanto a las clases artefactuales predominan en alta proporción los desechos de talla con un $91 \%$, y secundariamente los núcleos (5\%) y los artefactos formatizados (4\%). Una vez determinado el número de eventos de talla para ese componente, se procede a analizar cada uno de los nódulos múltiples aplicando el método no tipológico. Para ello, se seleccionan aquellos eventos que contienen desechos de talla. O sea que, pueden ser sólo desechos o una combinación de ellos con algún otro artefacto.

Del total de desechos $(n=187)$ se considera para el análisis el $25 \%(n=52)$ de la muestra, los cuales se hayan enteros. El promedio de fragmentación para la totalidad del conjunto es del 75\%. Una de las variables utilizadas es el tamaño, y en todos los eventos los tamaños más representados son el seis (muy grande) con el $27 \%$, el cuatro (mediano grande) con el $24 \%$, seguido del dos (17\%), tres (17\%) y cinco (15\%). En cambio el uno (muy pequeño) no está representado.

Teniendo en cuenta la cantidad de negativos previos de las lascas de todos los eventos, predominan aquellas que poseen dos negativos con el $23 \%(n=12)$, seguidas de las que poseen sólo uno, tres y cuatro. Es de destacar que hay un $15 \%(n=8)$ de lascas que no poseen ningún negativo.

Como dijimos anteriormente, para la aplicación del método no tipológico se seleccionan aquellos desechos enteros que corresponden a nódulos múltiples y que presentan las variables de tamaño y cantidad de negativos previos fundamentalmente. Entonces, mediante la fórmula, se procede a graficar las lascas de los eventos de talla. 


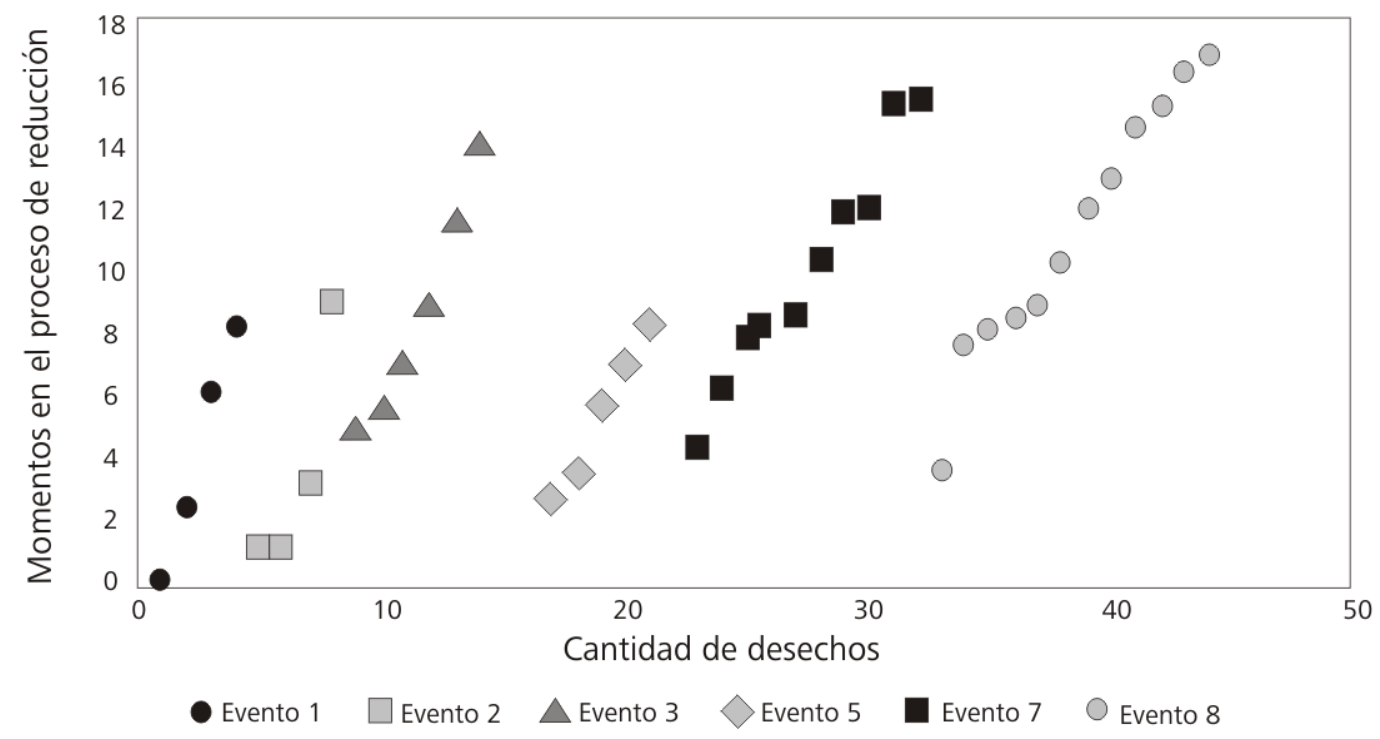

Figura 2. Representación de seis eventos de talla de ELS 2

Figure 2. Representation of six events from ELS 2

En la figura 2 se representan todos los nódulos múltiples de ELS 2 con la finalidad de comparar las diferentes secuencias de talla. Los eventos múltiples 1 y 2 (que contiene además un núcleo) poseen cuatro desechos de talla enteros y el tercero seis desechos enteros. Posteriormente se representan los eventos 5 (con 6 desechos y el cual contiene dos núcleos), el E7 con 10 desechos, y el E8 que incluye seis fragmentos de un mismo núcleo y siete instrumentos.

El componente I de ELS 3 contiene un total de 670 artefactos. A la unidad estratigráfica 11 pertenecen 61 artefactos (9\%), a la 14 le corresponden 200 artefactos (30\%) y, a la unidad estratigráfica 16, 397 artefactos (59\%). Con algunos de ellos (doce) no se pudo constatar la procedencia exacta de la unidad estratigráfica.

Al total del conjunto artefactual $(n=670)$ se le aplica el método de análisis nodular, en donde se pudieron
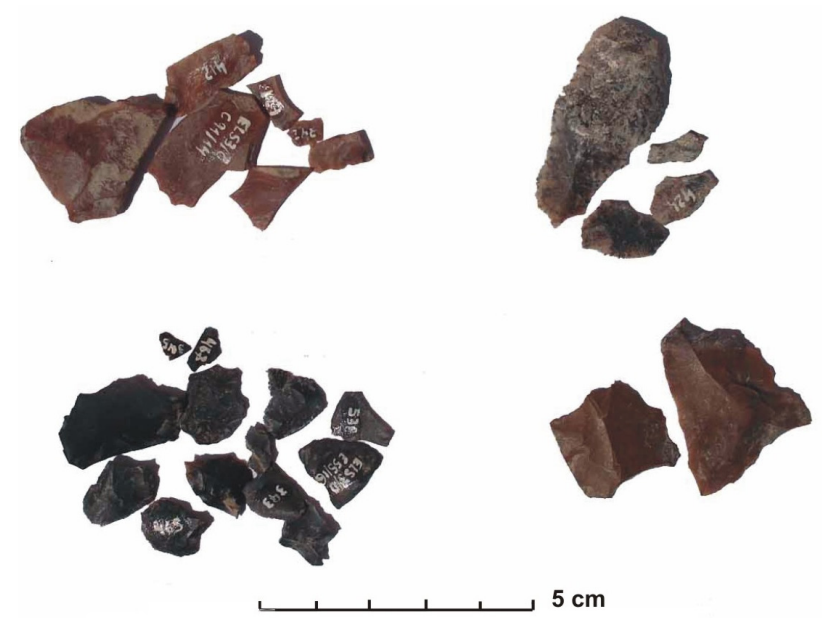

Figura 3. Cuatro nódulos múltiples de sílice Figure 3. Four silica multiple nodules establecer 384 nódulos, incluyendo simples y múltiples (Figura 3). 281 corresponden a los simples y 103 a los múltiples.

Para los nódulos múltiples se observan siete grupos de núcleos y desechos, 90 nódulos de desechos de talla, dos de bifaces y desechos y cuatro nódulos de instrumentos y desechos. La materia prima más representada es la roca silícea con un $92,3 \%(n=618)$ del total, seguida por el cuarzo con un 5,3\% $(n=36)$. El esquisto es una de las rocas menos abundante de la muestra con valores menores al $2 \%(n=13)$ y las materias primas indeterminadas constituyen menos de $1 \%(n=3)$.

En cuanto a las clases artefactuales predominan en alta proporción los desechos de talla con un 96,8\%, secundariamente los núcleos (1,9\%) y los bifaces $(0,9 \%)$. Además se registraron un raspador, una lasca con

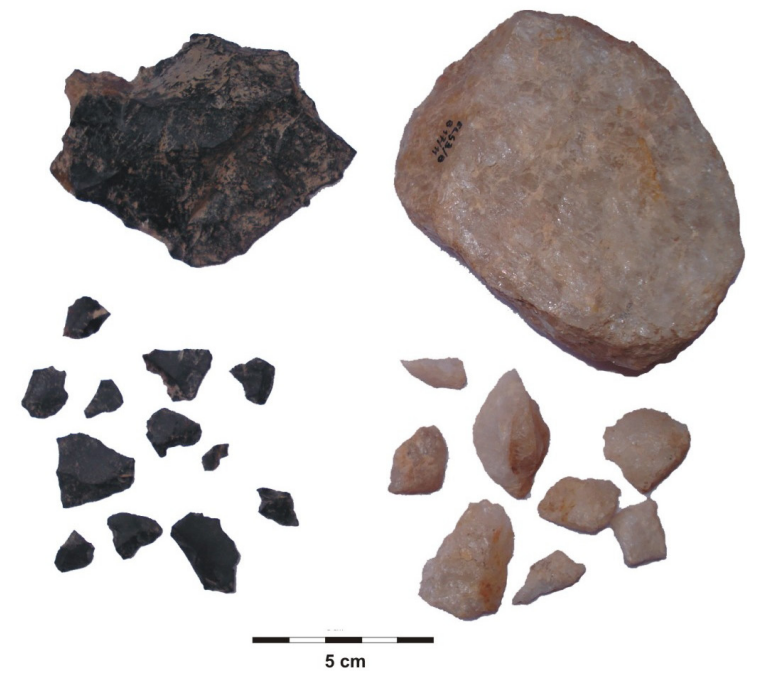

Figura 4. Dos nódulos múltiples de sílice (izquierda) y cuarzo (derecha) Figure 4. Two silica (left) and quartz (right) multiple nodules 
rastro complementario, un cepillo, dos percutores y dos artefactos pulidos (Figura 4).

En el análisis de los desechos de talla $(n=649)$ el $98 \%$ corresponden a lascas internas, con 134 pertenecientes a lascas de adelgazamiento (21\%). Del total de desechos sólo catorce presentan corteza, diez poseen terminación en charnela y 25 con evidencias de alteración térmica. El estado de fragmentación es del 70 \%. Los desechos enteros son 189, de los cuales encontramos 180 de roca silícea y 9 de cuarzo.

El tamaño más representado es el 2 que corresponde al pequeño con un total de $50 \%, 47 \%(n=94)$ en el caso de la sílice y un $3 \%(n=5)$ en el caso del cuarzo, seguido del tamaño 1 que corresponde al muy pequeño con un total del $25 \%$. Sólo la sílice cuenta con éste tamaño. El 3 (mediano pequeño) representa un $17 \%$ de total con $15 \%$ $(n=30)$ en la sílice y $2 \%(n=4)$ en el cuarzo. Los tamaños 4, 5 y 6 están en pequeñas proporciones del 6, 5 y $4 \%$ respectivamente. En el cuarzo no están representados.

Otra de las variables que se considera importante en este análisis es la cantidad de lascados previos en la cara dorsal. A un total de 188 desechos enteros se cuantificaron los negativos de lascado, obteniendo 180 para la sílice y ocho para el cuarzo. Del total, los más representados son los que presentan tres negativos con un $29 \%(n=55)$, seguido de dos con un $25 \%(n=47)$, cuatro con un $18 \%$ y con un negativo $16 \%$. Los que contienen cinco, seis, siete y ocho están escasamente representados.

Con la obtención de estos resultados se graficaron los nódulos múltiples representados en el Componente I de
ELS 3 (Figura 5). Los cinco eventos múltiples comparados (eventos 4, 16, 17, 50 y 56) poseen cuatro, cinco, seis y ocho desechos de talla. Los eventos 17 y 56 poseen además un fragmento de biface.

El componente II de ELS 3 contiene un total de 243 artefactos. De la unidad estratigráfica 2 pertenecen 162 artefactos (66 \%) y de la unidad estratigráfica 7, 80 artefactos (33\%). Sólo se presenta un caso sin procedencia exacta, aunque se pudo constatar que proviene de una de estas dos unidades estratigráficas.

Con la aplicación del método de análisis nodular se pudo establecer un total de 131 nódulos, entre simples y múltiples. 81 corresponden a nódulos simples y 50 a múltiples. En cuanto a los nódulos múltiples, se observan 47 nódulos de sólo desechos, uno de bifaces y desechos y dos nódulos de instrumentos y desechos (Sario 2011).

Con respecto a las materias primas, del total de 243 artefactos, el 90\% ( $n=218)$ son de materia prima sílice, el $9 \%$ de cuarzo $(n=22)$, y el $1 \%$ de calcedonia y rocas indeterminadas $(n=3)$. Entre las clases artefactuales se encuentran un artefacto compuesto, un biface, un raspador, una lasca retocada y 239 desechos de talla.

Los eventos que contienen desechos de talla pertenecen a lascas internas $(n=239)$. Las lascas de adelgazamiento son $40(17 \%)$, de las cuales tres presentan terminación en charnela. Además se pudieron observar ocho lascas con evidencias de alteración térmica. El estado de fragmentación del conjunto es del $77 \%$ y entre los desechos enteros $(n=54)$ encontramos 53 de roca silícea y uno de cuarzo.

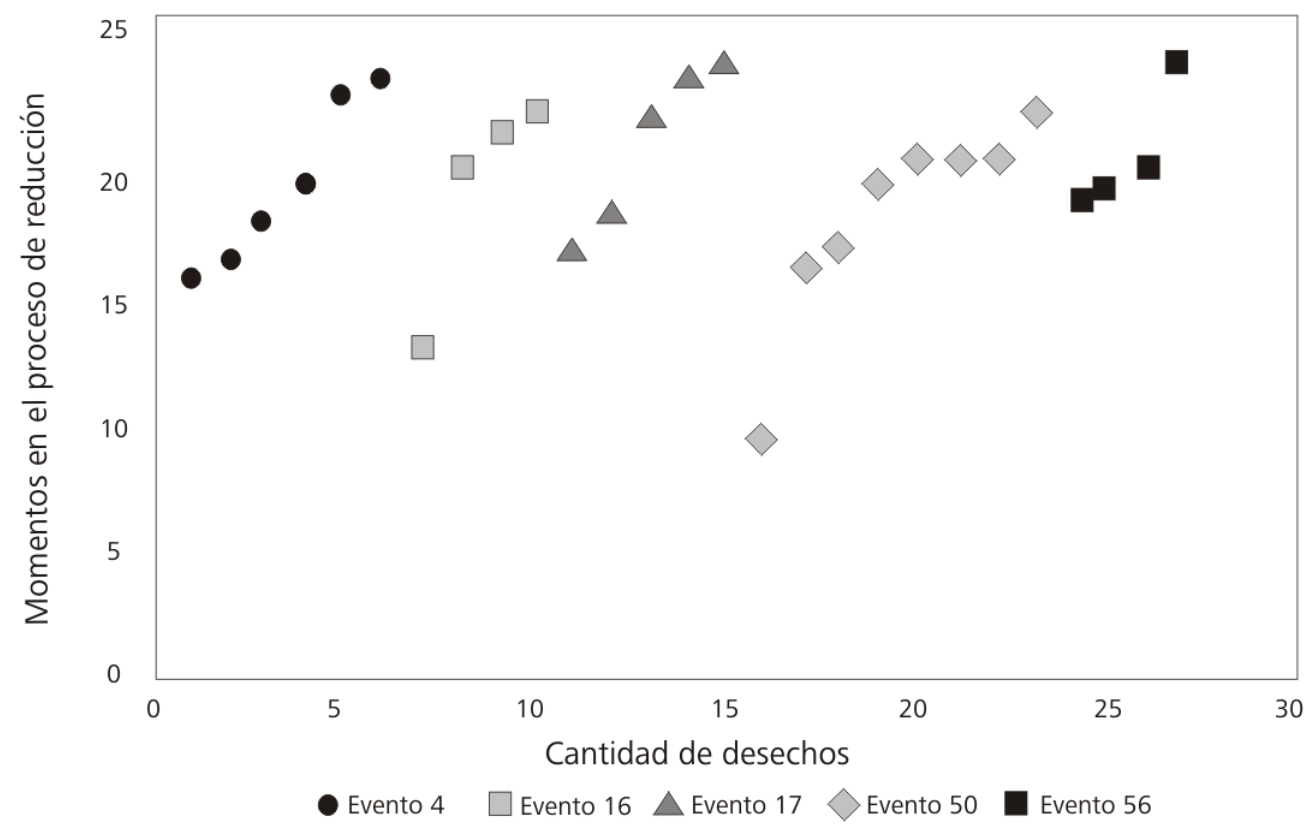

Figura 5. Representación de cinco eventos de talla del C I (ELS 3)

Figure 5. Representation of five events from C I (ELS 3) 


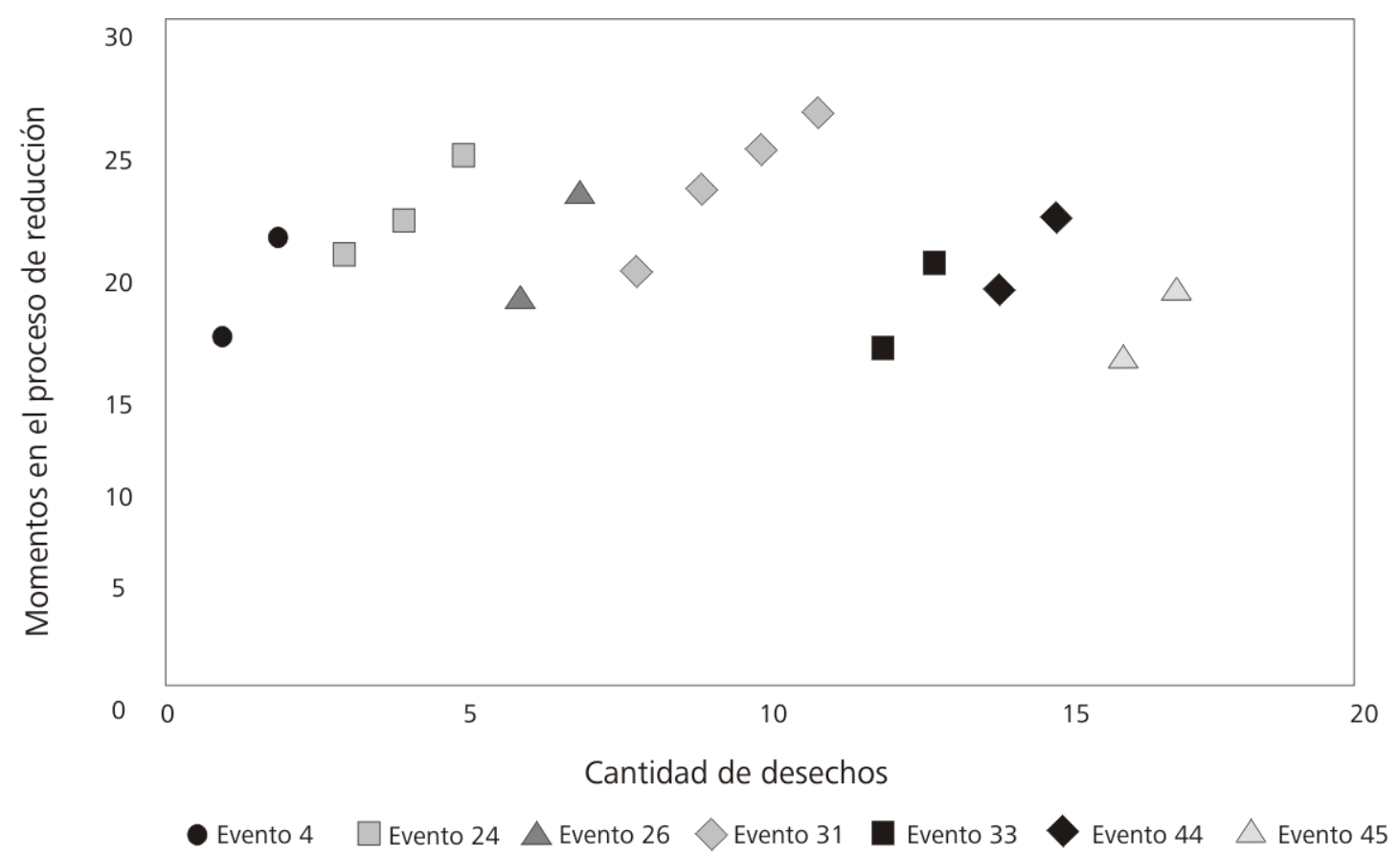

Figura 6. Representación de siete eventos de talla del C II (ELS 3)

Figure 6. Representation of seven events from C II (ELS 3)

El tamaño más representado es el tamaño 2 (pequeño) con un $32 \%$, seguido del tamaño 1 y 3 que corresponde al muy pequeño y mediano-pequeño con un total del $28 \%$ cada uno respectivamente. Los tamaños 4 y 5 están en pequeñas proporciones del $6 \%$ cada uno respectivamente. A un total de 51 desechos enteros de sílice se les pudo cuantificar los negativos de lascado. Los más representados son los que presentan 3 negativos con un $35 \%(n=18)$, seguido de 2 con un $31 \%(n=16)$, 4 con un $20 \%$ y con 1,5 y 6 negativos un $4 \%$. No se cuantificaron negativos de lascado en las demás materias primas.

Posteriormente se graficaron los nódulos múltiples representados en el Componente II de ELS 3 (Figura 6). Los siete eventos múltiples (eventos 4, 24, 26, 31, 33, 44 y 45) poseen dos, tres y cuatro desechos de talla. El evento 33 posee además un artefacto compuesto con forma base biface.

\section{Discusión}

Comparando los resultados del sitio 2 (cantera) y los dos componentes del sitio 3 podemos discutir algunas cuestiones: la primera, la materia prima dominante en los dos sitios es la roca silícea, con un menor ingreso a los sitios de otros tipos rocosos (Tabla 1).

En cuanto a las clases artefactuales hay un importante porcentaje de desechos de talla en los dos sitios, seguido por los núcleos y bifaces (Tabla 2). Lo que estaría indicando las actividades llevadas a cabo en los sitios, como los procesos de reducción y manufactura de instrumentos.

El sitio cantera (ELS 2) presenta una gran proporción de tamaños grandes entre sus desechos, con ausencia de los pequeños. Y en el sitio ELS 3 hay un mayor porcentaje de tamaños menores (Tabla 3). En la cantidad de negativos en la cara dorsal, si bien predominan los números menores en los dos sitios, hay ciertas diferencias como la ausencia de negativos en las lascas procedentes de ELS 2 y la presencia de números más elevados en ELS 3 (Tabla 4).

Entonces, se puede sostener con estos resultados que las primeras tareas de reducción eran realizadas en la cantera y los momentos intermedios y finales en el sitio taller. Sin embargo, lo interesante es que la combinación de los métodos tipológico y no tipológico estaría aportando un detalle más profundo de cómo fue la secuencia. Y que junto a la conformación de los eventos de talla de los sitios ELS 2 y ELS 3, se disponen en un mismo gráfico a modo de comparación (Figura 7).

En los primeros seis eventos de talla que corresponden a ELS 2, sus desechos se sitúan en la parte inferior del gráfico. Se puede observar que el evento 1 es el más inicial de todos los eventos, seguido por el evento 2. Los restantes, que son los eventos 3, 4, 5 y 6, contienen lascas que se disponen en un sector más intermedio de la secuencia, representando un momento más avanzado del proceso de reducción.

Todos los eventos de ELS 2 están situados en valores que van desde el momento 0 hasta el momento 17, indicando 


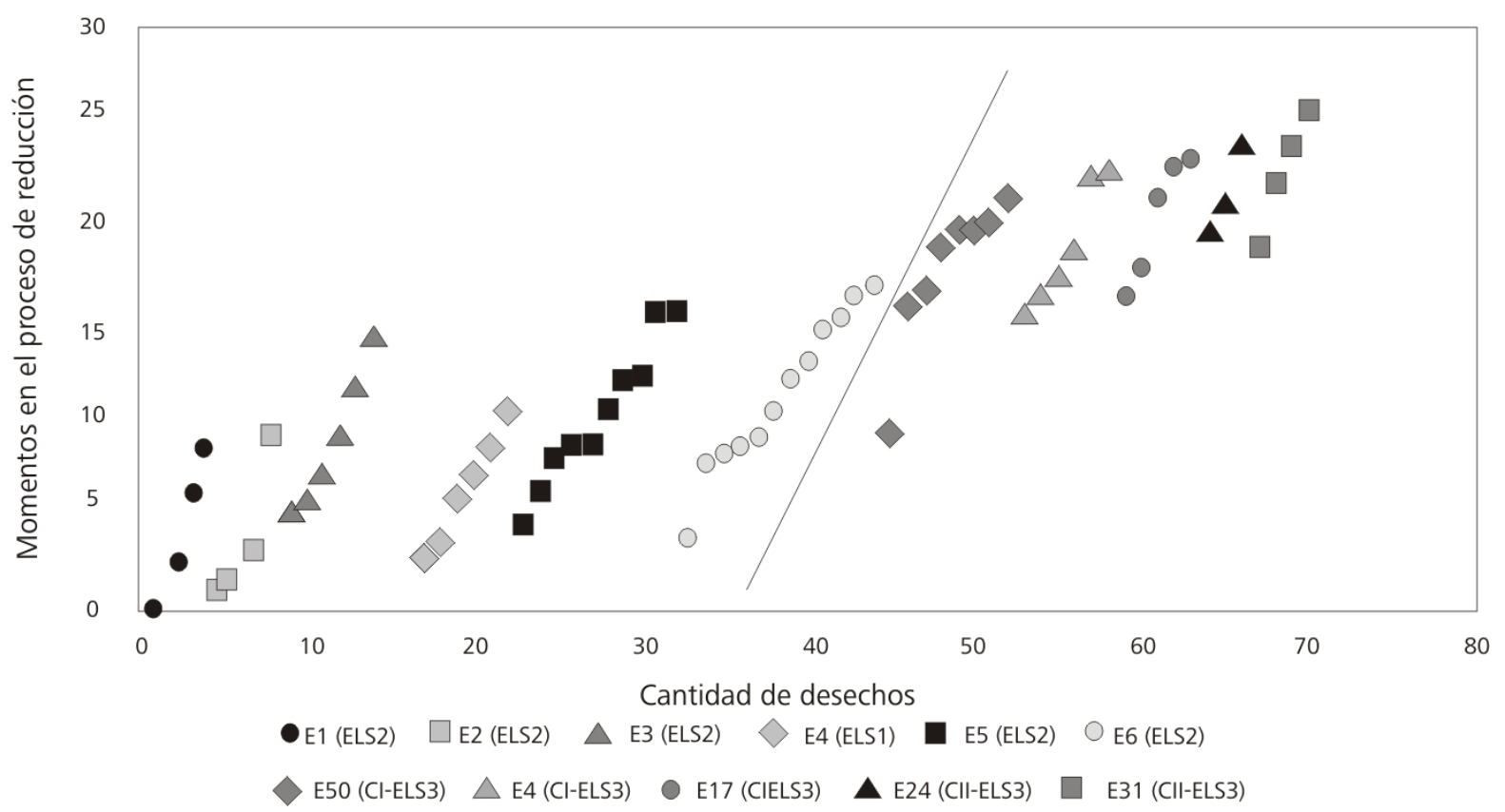

Figura 7. Representación de once eventos de talla de ELS 2 y los componentes I y II de ELS 3

Figure 7. Representation of eleven events from C I and II (ELS 3) and ELS 2

\begin{tabular}{lcccccc}
\hline & \multicolumn{2}{c}{ ELS 2 } & \multicolumn{2}{c}{ ELS 3 (C I) } & \multicolumn{2}{c}{$\operatorname{ELS} 3(\mathrm{C}$ II) } \\
\hline Materias primas & $\mathrm{N}$ & $\%$ & $\mathrm{~N}$ & $\%$ & $\mathrm{~N}$ & $\%$ \\
Sílice & 203 & 99 & 618 & 92,3 & 218 & 90 \\
Cuarzo & 1 & 1 & 36 & 5,3 & 22 & 9 \\
Esquisto & - & - & 13 & 1,9 & - & - \\
Calcedonia & - & - & - & - & 2 & 1 \\
Indeterminadas & - & - & 3 & 0,44 & 1 & 0 \\
Total & 204 & 100 & 670 & 100 & 243 & 100 \\
\hline
\end{tabular}

Tabla 1. Materias primas de los sitios ELS 2 y ELS 3

Table 1. ELS2 and ELS3 sites raw materials.

\begin{tabular}{lcccc}
\hline Clases artefactuales & ELS 2 & $\begin{array}{l}\text { ELS 3 } \\
\text { (C I) }\end{array}$ & $\begin{array}{l}\text { ELS 3 } \\
\text { (C II) }\end{array}$ & Total \\
\hline Núcleos & - & 6 & 1 & 7 \\
Bifaces & 1 & 1 & 1 & 2 \\
Raspadores & - & 1 & - & 1 \\
Cepillos & - & 2 & - & 2 \\
Artefactos pulidos & - & 2 & - & 2 \\
Percutores & - & - & 1 & 1 \\
Artefactos compuestos & 1 & 1 & - & 1 \\
Lascas con rastros complementarios & 5 & - & 1 & 1 \\
Lascas con retoque & 187 & 649 & 239 & 1074 \\
Desechos de talla & 204 & 670 & 243 & 1117 \\
Total & & & & \\
\hline
\end{tabular}

Tabla 2. Clases artefactuales de los sitios ELS 2 y ELS 3

Table 2. ELS 2 and ELS 3 sites artifact classes

\begin{tabular}{|c|c|c|c|c|c|c|}
\hline \multirow[b]{3}{*}{ Tamaño } & \multicolumn{6}{|c|}{ DESECHOS ENTEROS } \\
\hline & \multicolumn{2}{|c|}{ ELS 2} & \multicolumn{2}{|c|}{ C I (ELS 3) } & \multicolumn{2}{|c|}{ C II (ELS 3) } \\
\hline & $\mathrm{N}$ & $\%$ & $\mathrm{~N}$ & $\%$ & $\mathrm{~N}$ & $\%$ \\
\hline 1 & - & - & 50 & 25 & 15 & 28 \\
\hline 2 & 9 & 17 & 99 & 50 & 18 & 32 \\
\hline 3 & 9 & 17 & 34 & 17 & 15 & 28 \\
\hline 4 & 12 & 24 & 6 & 3 & 3 & 6 \\
\hline 5 & 8 & 15 & 5 & 3 & 3 & 6 \\
\hline 6 & 14 & 27 & 4 & 2 & - & - \\
\hline Total & 52 & 100 & 198 & 100 & 54 & 100 \\
\hline
\end{tabular}

Tabla 3. Tamaño de los desechos líticos de los sitios ELS 2 y ELS 3 Table 3. Lithic debitage size from sites ELS2 and ELS3

\begin{tabular}{ccccccc}
\hline \multicolumn{7}{c}{ DESECHOS ENTEROS } \\
\hline DSC & ELS 2 & $\%$ & C I (ELS 3) & $\%$ & C II (ELS 3) \\
S/DSC & 8 & 15 & - & - & - & $\%$ \\
1 & 11 & 21 & 31 & 16 & 2 & 4 \\
2 & 12 & 23 & 47 & 25 & 16 & 31 \\
3 & 10 & 19 & 55 & 29 & 18 & 35 \\
4 & 10 & 19 & 34 & 18 & 10 & 20 \\
5 & 1 & 2 & 10 & 5 & 2 & 4 \\
6 & - & - & 9 & 5 & 2 & 4 \\
7 & - & - & - & - & 1 & 2 \\
8 & - & - & 2 & 1 & - & - \\
Total & 52 & 100 & 188 & 100 & 51 & 100 \\
\hline
\end{tabular}

Tabla 4. Negativos en la cara dorsal de los desechos líticos en los sitios ELS 2 y ELS 3

Table 4. ELS 2 and ELS3 lithic debitage dorsal scares. 
momentos iniciales de la secuencia de talla. Los tres subsiguientes, los eventos 50, 4 y 17, que corresponden al componente I de ELS 3, se disponen en un sector medio y superior del gráfico, entre valores que van mayormente desde el 15 al momento 23. Las lascas de los dos últimos eventos, 24 y 31, que pertenecen al C II de ELS 3, se sitúan entre los momentos 18 y 26 , representando un sector más superior en el gráfico. Lo que permite inferir, para estos últimos cinco eventos de ELS 3, momentos de producción medios a finales. Siendo los eventos del C II un poco más avanzados en la trayectoria de producción que en el C I.

Las ausencias de lascas, dentro de cada una de las secuencias, pueden ser interpretadas como una selección de las mismas para ser utilizadas para la posterior confección de instrumentos, o simplemente pudieron no estar representadas porque se encuentran fracturadas. Otra opción, es que se hallen en un área en donde no hubo recolección o excavación.

\section{Consideraciones Finales}

El registro arqueológico lítico de las sociedades prehispánicas del área de la Sierra de la Estanzuela puede ser analizado utilizando un modelo experimental que considera la producción lítica como proceso un continuo, siguiendo las propuestas de Ingbar et al. (1989).

El método no tipológico permite posicionar una lasca en la secuencia de producción, y contemplar si las secuencias son completas o discontinuas. De esta manera, con el modelo cuatro planteado por los autores se comparan los diferentes momentos dentro de la trayectoria de producción, resultando ser adecuada para este contexto. Sin embargo, en la actualidad se están buscando generar nuevos modelos en función de las propias experimentaciones con rocas locales.

Con estos resultados, se puede observar un uso diferencial del espacio, en el que se estuvieron realizando las reducciones iniciales en la cantera (ELS 2). En este sitio no se completó el proceso, las formas base fueron trasladadas hacia ELS 3 u otras locaciones para continuarlo. Y aunque no se han hallado los sitios de utilización de los instrumentos, se puede observar un uso del espacio y de la producción tecnológica basada en la discontinuidad. Asimismo, los mismos métodos fueron aplicados en los conjuntos líticos de sitios tempranos de Tandilia en la región pampeana y en Cueva Fell de la región patagónica con resultados similares (Cattáneo y Flegenheimer 2008; Flegenheimer y Cattáneo 2010).

Para finalizar, a pesar de que no se pueden comparar cronológicamente los dos sitios, sí se puede plantear que las prácticas de los grupos humanos del pasado pueden ser compartidas y heredadas a lo largo del tiempo. Por ello, se plantea que los grupos prehispánicos que habitaron la Sierra de la Estanzuela compartían prácticas similares con otras sociedades humanas de otras regiones, como parte de una red de relaciones extraterritoriales (Laguens 2009).

Córdoba, 1 de junio de 2012

\section{Agradecimientos}

A todos los que cooperaron en las tareas de campo, estudiantes de la Universidad Nacional de Córdoba, compañeros del Museo de Antropología (FFyH, UNC) y colaboradores de Córdoba y San Luis. A los directores de los proyectos CONICET, FONCyT, SECyT (Cba.) Roxana Cattáneo, Andrés Laguens y Darío Demarchi que guiaron esta investigación. También agradezco muy especialmente a Hugo Nami por sus comentarios y sugerencias.

\section{Bibliografía}

Ahler, S.A. 1989. Mass analysis of flaking debris: studying the forest rather than the trees. Alternative Approaches to Lithic Analysis. Henry, D.O., Odell, G.H. (Eds.). Archaeological Papers of the American Anthropological Association Number 1, pp. 85-118.

Amick, D.S. y R.P. Mauldin. 1997. Effects of raw material on flake breakage patterns, Lithic Technology 22: 18-32.

Amick, D. S., R. P. Mauldin y L. R. Binford. 1989. The Potentials of Experiments in Lithic technology. En Experiments in Lithic Technology, Editado por D. S. Amick and R. P. Mauldin, pp. 1-14. British Archaeological Reports International Series 528, Oxford.

Aschero, C. 1983. Ensayo para una clasificación morfológica de artefactos líticos aplicada a estudios tipológicos comparativos. Revisión. Cátedra de Ergología y Tecnología de la Facultad de Filosofía y Letras de la U.B.A. MS en DIPA, IMHICIHU, CONICET.

Bellelli, C., A. Guraieb y J. García. 1985-87. Propuesta para el análisis y procesamiento por computadora de desechos de talla lítica (DELCO). Arqueología contemporánea 2: 36-53. Buenos Aires.

Bradbury, A. P. y P. J. Carr. 1995. Flake tipologies and alternative approches: an experimental assessment. En Lithic Technology 20 (2): 100-115.

Cattáneo, G. R. 2005. Tecnología lítica en la localidad arqueológica Piedra Museo (Santa Cruz, Argentina). Relaciones de la Sociedad Argentina de Antropología XXX: 79-103.

Cattáneo, G. R. 2006. Tecnología Lítica del Pleistoceno Final/Holoceno Medio. Un Estudio de los CazadoresRecolectores de la Patagonia Austral (Argentina). BAR Internacional Series 1580. Oxford. 
Cattáneo, G. R. y N. Flegenheimer. 2008. Análisis comparativo de desechos de talla, en contextos tempranos de Chile y Argentina. Trabajo presentado en el VII Jornadas de Arqueología de la Patagonia. Ushuaia, Tierra del Fuego.

Charlin, J. 2009. Estrategias de aprovisionamiento y utilización de las materias primas líticas en el campo volcánico de Pali Aike (provincia de Santa Cruz, Argentina). BAR Internacional Series 1901. Oxford.

Eerkens J. W., J. R. Ferguson, M. D. Glascock, C. E. Skinner y A.W Sharon. 2007. Reduction strategies and geochemical characterization of lithic assemblages: a comparasion of three studies from western north America. American Antiquity, 72(3), pp. 585-597.

Escola, P. 2000. Tecnología lítica y Sociedades Agropastoriles Tempranas. Tesis de Doctorado inédita, Facultad de Filosofía y Letras, Universidad de Buenos Aires.

Espinoza, S. 1998. Desechos de talla: tecnología y uso del espacio en el Parque Nacional Perito Moreno (Santa Cruz, Argentina). Anales del Instituto de la Patagonia. Serie Ciencias Humanas 26: 153-168.

Flegenheimer, N. 1991. La Liebre, un sitio cantera-taller. Boletín del Centro 2: 58-64.

González de Bonaveri, M. I y Horovitz, L. 1991. Desechos de talla del Sitio L.G.I, Partido de Chascomús, Prov. de Buenos Aires. Shincal 3, (2): 52-63 Catamarca.

Flegenheimer, N. y Cattáneo, R. 2010. Producción lítica a través del espacio: análisis comparativo en contextos del Pleistoceno final/ Holoceno temprano de Chile y Argentina. Resúmenes del V Simposio Internacional El Temprano en América: a cien años del debate AmeghinoHrdlicka. La Plata, Buenos Aires.

Ingbar, E. 1994. Lithic material selection and technological organization. En The organization of North American Prehistoric Chipped Stone Technologies, editado por J. Carr. pp 45-56. International Monographs in Prehistory Archaeological Series 7.

Ingbar, E., M. Larson y B. Bradley. 1989. A non typological approach to débitage analysis. Experiments in lithic technology. D. Amick y R. Mauldin (eds.). pp. 117-136. British Archaeological Reports - Series 528.

Laguens, A. 2009. De la diáspora al laberinto: Notas y reflexiones sobre la dinámica relacional del poblamiento humano en el centro-sur de Sudamérica. Arqueología suramericana 5 (1): 42-67.

Laguens, A., R. Cattáneo, E. Pautassi y G. Sario. 2009.
Poblamiento humano temprano en las Sierras de San Luis: Estancia La Suiza. en Martini, Y.; Pérez Zavala, G. y Aguilar, Y. (compiladoras): Las sociedades de los paisajes áridos y semiáridos del centro-oeste argentino. Río Cuarto, Editorial de la Universidad Nacional de Rio Cuarto, pp. 41-60.

Laguens, A., E. Pautassi, G. Sario y R. Cattáneo. 2007. Fishtail Projectil Points from Central Argentina. Current Research in Pleistocene 24: 55-57.

Larson, M. 2004. Chipped Stone Aggregate Analysis in Archaeology. Aggregate Analysis in Chipped Stone: 3-17. C. T. Hall y M. L. Larson (eds.). University of Utah.

Larson, M. y M. Kornfeld. 1997. Chipped stone nodules: theory, method and examples. Lithic Technology 22 (1): 4-18.

Larson, M. L y Finley, J. B. 2004. Seeing the Trees but Missing the Forest: Production Sequences and Multiple Linear Regression. Aggregate Analysis in Chipped Stone: 95-113. C. T. Hall y M. L. Larson (eds.). University of Utah.

Magne, M. P. R. 2001. Debitage analysis as a scientific tool for archaeological knowledge. Lithic Debitage: Context, Form, Meaning. Andrefsky Jr., W. (Ed.). The University of Utah Press, pp. 21-31.

Nami, H. G. 1997-1998. Arqueología experimental, talla de piedra contemporánea, arte moderno y técnicas tradicionales: observaciones actualísticas para discutir estilo en tecnología lítica. Relaciones de la Sociedad Argentina de Antropología XXII-XXIII: 363-388. Buenos Aires.

Nami, H. G. 2007. Epistemología y consideraciones sobre arqueología y tecnología lítica experimental. Publicación realizada para el Taller de Arqueología y Tecnología lítica dictado en la Universidad de San Cristóbal de Huamanga, Ayacucho, Perú. Pp. 1-63. Buenos Aires.

Nami, H. G. y C. Bellelli. 1994. Hojas, experimentos y análisis de desechos de talla. Implicaciones arqueológicas para la Patagonia Centro-Septentrional. Cuadernos del Instituto Nacional de Antropología y Pensamiento Latinoamericano 15: 199-223.

Prentiss, W. 1998. The Reliability and Validity of a Lithic Debitage Typology: Implications for Archaeological Interpretation. American Antiquity, 63 (4): 635-650. Sario, G. 2007. Análisis morfo-tecnológico de los materiales líticos del sitio Estancia La Suiza 3 (provincia de San Luis). En Resúmenes ampliados del XVI Congreso Nacional de Arqueología Argentina. Revista Pacarina III: 497-500. Jujuy.

Sario, G. 2009. Estancia La Suiza 3 (provincia de San Luis): 
un estudio de la tecnología lítica. La Zaranda de Ideas, Revista de Jóvenes Investigadores en Arqueología 5: 45-64. Buenos Aires.

2011. Poblamiento humano en la provincia de San Luis: una perspectiva arqueológica a través del caso de la organización de la tecnología en Estancia La Suiza. Tesis doctoral inédita, Facultad de Filosofía y Humanidades, Universidad Nacional de Córdoba.

Shott, M. y P. Sillitoe. 2005. Use life and curation in New Guinea experimental used flakes. Journal of
Archaeological Science 32: 653-663.

Sullivan, A. P y C. Rozen. 1985. Debitage Analysis and Archaeological Interpretation. American Antiquity 50: 755-779.

Valverde, F. 2003. Análisis de los desechos líticos de la ocupación inicial del sitio Cueva Tixi (provincia de Buenos Aires): cadena operativa de producción y técnicas de talla tempranas. Relaciones de la Sociedad Argentina de Antropología XXVIII: 185-203. Buenos Aires. 